\title{
Encoding Human Sexual Chemosensory Cues in the Orbitofrontal and Fusiform Cortices
}

\author{
Wen Zhou and Denise Chen \\ Psychology Department, Rice University, Houston, Texas 77005
}

\begin{abstract}
Chemosensory communication of affect and motivation is ubiquitous among animals. In humans, emotional expressions are naturally associated with faces and voices. Whether chemical signals play a role as well has hardly been addressed. Here, we use functional magnetic resonance imaging to show that the right orbitofrontal cortex, right fusiform cortex, and right hypothalamus respond to airborne natural human sexual sweat, indicating that this particular chemosensory compound is encoded holistically in the brain. Our findings provide neural evidence that socioemotional meanings, including the sexual ones, are conveyed in the human sweat.
\end{abstract}

Key words: olfactory; chemosensory; fMRI; emotion; sex; human

\section{Introduction}

It is well known that many animals communicate salient information including sexual interest through changes in chemosignals produced from the body and skin (Wyatt, 2003). Our understanding of the function of human chemosignals, however, is still limited. One fruitful approach that links social behavior with chemosignals has been the study of the putative sex pheromone androstadienone (PSP). PSP is an odorous steroid that is found in greater amount in men than in women (Brooksbank et al., 1972). In women, it exerts a positive effect on mood and cognition (Jacob and McClintock, 2000; Lundström et al., 2003; Bensafi et al., 2004; Wyart et al., 2007) and heightens sympathetic nervous system arousal (Jacob et al., 2001a; Bensafi et al., 2004; Wyart et al., 2007). In addition, it is distinguished from common household smells in terms of women's neural responses, as revealed by several positron emission tomography (PET) studies (Jacob et al., 2001b; Savic et al., 2001, 2005; Gulyás et al., 2004).

In the present experiment, we chose to directly study natural human sexual sweat, which people encounter in natural settings. The human sweat, consisting of steroids [including PSP (see Materials and Methods)] and acids (Zeng et al., 1991; Labows and Preti, 1992), is a conglomerate of secretions from the sebaceous, eccrine, and apocrine glands that respond to emotion (Labows and Preti, 1992). Considered a social chemosignal, sweat has been shown in behavioral studies to convey individual identity (Russell, 1976), reproductive state (Stern and McClintock, 1998), and possibly affect (happiness, fear, anxiety) of the sweat donors (Chen and Haviland-Jones, 2000; Pause et al., 2004; Chen et al., 2006; Zhou and Chen, 2009). In the domain of individual iden-

Received July 7, 2008; revised 0ct. 29, 2008; accepted Nov. 24, 2008.

This work was supported in part by National Institutes of Health Grant R03 DC4956. We thank Jay Gottfried, Wen Li, Michael Beauchamp, Darcy Burgund, Phil Burton, and Tony Ro for useful discussions, and Ping Hou and Gang Chen for helpful assistance with the MRI pulse sequence and the AFNI ANCOVA program, respectively.

Correspondence should be addressed to Denise Chen, Psychology Department MS25, Rice University, 6100 Main Street, Houston, TX 77005. E-mail: xdchen@rice.edu.

DOI:10.1523/JNEUROSCI.3148-08.2008

Copyright $\odot 2008$ Society for Neuroscience $\quad 0270-6474 / 08 / 2814416-06 \$ 15.00 / 0$ tity, a recent PET study suggests that the brain also differentiates between sweat from familiar and unfamiliar women (Lundström et al., 2008).

To examine how the brain functionally processes human sexual sweat, we performed the first functional magnetic resonance imaging (fMRI) study of naturally produced human social chemosensory compounds, using a slow event-related design that enabled us to examine the temporal dynamics of the responses. We examined the sweat of sexual arousal in comparison with two other social chemosensory compounds (PSP and the sweat of neutrality) and a nonsocial smell [phenyl ethyl alcohol (PEA)]. We were able to overcome the inherent variation in sensory differences typically associated with olfactory stimuli by using ANCOVA to statistically control for activities associated with odor intensity and pleasantness. Finally, we performed functional connectivity analyses to probe the origins of the observed activation patterns.

We tested 19 healthy female subjects who inhaled each type of olfactory stimuli twice (in nonconsecutive order) per run, over a course of five runs. The subjects followed visual prompts to inhale ("I") and exhale ("O"), rated the intensity/pleasantness after each olfactory presentation, and described what each smelled like at the end of the study [see Materials and Methods for details, supplemental Figure S1 (available at www.jneurosci.org as supplemental material) for an illustration of the procedure, Fig. 1 for intensity/pleasantness ratings, and Table 1 for verbal descriptions]. We monitored respiration on 18 of the subjects.

\section{Materials and Methods}

Sweat collection. From $2 \mathrm{~d}$ before the experiment until the end of the experiment, 20 heterosexual male donors in a larger study refrained from using deodorant/antiperspirant/scented products, and used scent-free shampoo/conditioner, soap, and lotion provided by the experimenter. They reported to have experience with watching sexually explicit videos, and signed informed consent before participation. Subjects kept a 4 “ $\times$ 4" pad (rayon/polyester for maximum absorbance) in each armpit while they watched 20-min-long video segments intended to produce the emotions of sexual arousal (sexual intercourse between heterosexual couples) 

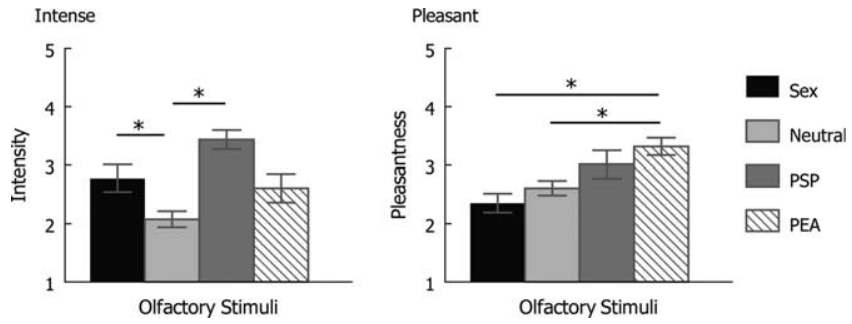

Figure 1. Mean intensity and pleasantness ratings. There are four types of olfactory stimuli, and SE bars are shown. For intensity, 1 refers to no smell, 2 little smell, 3 moderate smell, 4 quite a bit smell, and 5 strong smell. For pleasantness, 1 refers to very unpleasant, 2 unpleasant, 3 neutral, 4 pleasant, and 5 very pleasant. Sex, Sexual sweat; Neutral, neutral sweat. Sexual sweat and PSP were perceived to be more intense than neutral sweat; PEA was perceived to be more pleasant than sexual sweat and neutral sweat. ${ }^{*} p<0.05$, Bonferroni corrected.

and neutrality (educational documentaries), respectively. Meanwhile, their skin conductance was recorded using $\mathrm{Ag} / \mathrm{AgCl}$ electrodes filled with isotonic electrolyte (BIOPAC Systems) attached to the palmar area of the nondominant hand. New pads were used for each segment. The sequence of segment presentation was counterbalanced. A 5 min segment (of the same emotional content as the 20 min segment) preceded each $20 \mathrm{~min}$ segment, serving as an emotional transition. Only pads worn during the 20 min segments were used in the fMRI study. Once collected, sweat pads were stored at $-80^{\circ} \mathrm{C}$ until subsequent testing. Over the course of the 20 min video segments, donors experienced greater arousal (measured by skin conductance) while watching erotic videos than while watching neutral videos $\left(t_{(14)}=2.705, p=0.017\right.$, excluding five donors whose data could not be used because of failure of electrodes and other technical difficulties). Three healthy, male nonsmokers (aged 26, 29, and 29 years) were subsequently selected for the current study mainly because of their higher level of the self-reported sexual arousal (Fig. 2).

$f M R I$ participants. We recruited only women for their superior sense of smell (Brand and Millot, 2001) and sensitivity to emotional signals (Broday and Hall, 2000). Twenty right-handed females (mean age $=23.4$ years; $\mathrm{SE}=0.89$ years) were selected from a group of 42 women on the basis that they reported to have no rhinal disorders or neurological diseases, and that they showed superior olfactory sensitivity to PSP (mean $=$ $3.0 \mu \mathrm{M}, \mathrm{SE}$ in binary dilution step $=0.48)$ and PEA $($ mean $=0.00042 \%$, $\mathrm{SE}$ in binary dilution step $=0.61$ ). They either were in a heterosexual relationship or had been in one within the previous year. They were not on hormone contraceptives, and were tested during the periovulatory phase of their menstrual cycles $(10-27 \mathrm{~d}$, on average $17 \mathrm{~d}$, from the onset of their last period). All gave written consents for participation. One subject's data were excluded from further analysis because of excessive head movement. Subjects were informed that the study was on brain activations to natural compounds. They were blind to the nature of the smells used in the experiment.

Olfactory stimuli. Three types of social chemosensory compounds were used. One was the pooled sweat of sexual arousal obtained from the three heterosexual male donors (sexual sweat), another was the sweat from the same men but under neutral emotions (neutral sweat), and the third was the putative sex pheromone PSP (Steraloids; $916 \mu \mathrm{m}$ in propylene glycol, $1 \mathrm{ml}$ ). PEA (Sigma-Aldrich; $0.03 \%$ in propylene glycol, 1 $\mathrm{ml}$ ) was used as a nonsocial control. The concentrations of PSP and PEA were chosen based on a separate panel of 10 female judges who were told to best match their intensity and pleasantness to those of the sexual sweat. All smells were presented on nylon/polyester blended pads. We estimated the amount of PSP in our sweat sample to be $<0.0055 \mu \mathrm{mol}$, calculated as PSP concentration in the underarm skin extract (Gower et al., 1994) $\left(17.9 \mathrm{pmol} / \mathrm{cm}^{2}\right)$ times pad size $(10.16 \times 10.16 \mathrm{~cm})$ times donor number (3).

Data acquisition. Data were collected using a 3T Philips Intera and asymmetric spin echo pulse sequence (Stables et al., 1998) with echo time $=31 \mathrm{~ms}$, repetition time $(\mathrm{TR})=2000 \mathrm{~ms}$, field of view $=240 \times 240$ $\mathrm{mm}^{2}$, matrix $=128 \times 128$, and number of slices $=28$. The in-plane resolution was $1.875 \times 1.875 \mathrm{~mm}^{2}$. Slice thickness $=3.75 \mathrm{~mm}$. Four dummy scans were conducted at the beginning of each run to allow the magnetization to reach magnetic equilibrium. A T1-weighted anatomical volume (three-dimensional magnetization-prepared rapidacquisition gradient echo; $1 \times 1 \times 1 \mathrm{~mm}^{3}$ resolution) was also acquired for each subject for normalization and visualization of the functional data. Slices were acquired using oblique orientation $30^{\circ}$ to the anterior commissure-posterior commissure line (Sobel et al., 1997). Respiration was monitored with the scanner's built-in sensor that was taped on subjects' diaphragms and recorded using Biopac MP150 (Biopac Systems).

$f M R I$ procedure. Each olfactory presentation lasted for $12 \mathrm{~s}$ consisting of inhalation (4s), exhalation (4s), and inhalation (4s). Each olfactory stimulus was presented twice per run over a course of five runs in a semirandomized manner via a nonferric, computer-controlled, sixchannel MRI-compatible olfactometer (Mindware Technologies) adapted from the study by Lorig et al. (1999). Inhalations and exhalations were prompted respectively by "I" and "O" signs on the screen so that breathing can be synchronized with smell delivery. There was a $28 \mathrm{~s}$ interval between two smell presentations, during which only air was delivered and subjects followed "I" and "O" prompts as usual. Immediately after each smell presentation, subjects rated either the intensity or the pleasantness of the smell on a 1-5 scale using a response pad attached to their right hand. The order in presenting the intensity and pleasantness questions was counterbalanced across the subjects and the scans. After the scans, 16 of the 19 subjects smelled the same olfactory stimuli presented through the olfactometer and described what each smelled like. Before the scans, all subjects had been trained to breathe according to the rhythm of the visual prompts. The fMRI session took $\sim 1$ h to finish.

$f M R I$ data analysis. Functional data were analyzed using AFNI (Cox, 1996). After image preprocessing, the fMRI data were analyzed using the general linear model (GLM). Regressors of interest, each corresponding to the time points when subjects inhaled a particular olfactory stimulus (each $12 \mathrm{~s}$ presentation consisted of two such inhalations), were convolved with the gamma function. Drifting effect, head motion, and questions (when subjects were prompted to rate the intensity or pleasantness of an olfactory stimulus, convolved with the gamma function) were also included in the GLM. The parameter estimates (betas) for each olfactory stimulus were calculated for all brain voxels and entered in the secondlevel ANCOVA together with the average intensity and pleasantness ratings for each olfactory stimulus from each subject. ANCOVA was used to analyze activations to the social chemosensory compounds (sexual sweat, neutral sweat, PSP) with the nonsocial control smell (PEA) as the baseline. The use of ANCOVA was important because it allowed us to normalize the inherent intensity and pleasantness variations (Fig. 1) in the olfactory stimuli. The average percentage signal changes within the activated regions were then extracted (assuming the shape of the hemodynamic response) for each olfactory condition from each subject. The impulse response function after the inhalation of each olfactory stimulus (each olfactory regressor corresponds to the onsets of inhaling an olfactory stimulus; each $12 \mathrm{~s}$ presentation consisted of two such inhalations) was estimated with AFNI 3dDeconvolve using a minimum time lag $=0$ $\mathrm{TR}$ and a maximum time lag $=7 \mathrm{TR}$ (without assuming the shape of the hemodynamic response). In our voxelwise analysis, we used alpha probability simulation (AlphaSim) and applied small volume correction to the three heavily documented olfactory regions [the piriform, amygdala, and orbitofrontal cortex (OFC) (Sobel et al., 2000; Gottfried et al., 2002a)] and the hypothalamus. The AlphaSim program uses Monte Carlo simulation. The probability of a false-positive detection for an region of interest is determined from the frequency count of cluster sizes based on the combination of individual voxel probability thresholding and minimum cluster size thresholding [for details, please refer to Ward (2000)]. For the rest of the brain, we used an uncorrected $p<0.0005$, corresponding to $Z>3.48$ in combination with a cluster size $>40 \mathrm{~mm}^{3}$.

To assess the functional connectivity involved in processing human sexual sweat, we first performed a simple correlation analysis. Piriform and amygdala were anatomically located according to Talairach coordinates and human brain atlases (Talairach and Tournoux, 1988; Mai et al., 1997). Variance explained by regressors other than sexual sweat, including baseline, drifting effects, head motion, and responding to the intensity/pleasantness questions, as well as neutral sweat, PSP, and PEA, were 
removed from the original time series for each subject to make the signals immune to any undesirable effect. This was done for each subject by (1) selecting the regression coefficients and the $\mathrm{X}$ matrix columns corresponding to all the regressors except sexual sweat from the GLM regression analysis described above; (2) deriving a fit time series dataset from (1) using AFNI program 3dSynthesize; and (3) subtracting (2) from the original time series. The subjects' resulting EPI data in time series were converted to the Talairach space. Time series were extracted from the voxel with the peak $t$ value in the OFC

(Talairach coordinates $33,40,-1$ ) and the voxel with the peak $t$ value in the right fusiform (Talairach coordinates $35,-51,-7$ ), respectively. Each was then respectively used as the covariate of interest in a wholebrain linear regression analysis in which global signal variation resulting from physiological noises was set as an orthogonal regressor. Fisher's $Z$ transformation $z=(1 / 2) \ln [(1+r) /(1-r)]$ was applied to the correlation coefficients to reduce skewness and to normalize the sampling distribution. The $z$ scores were entered into a second-level random-effects analysis to determine the brain areas whose hemodynamic responses (HRs) significantly covaried with the seed HR across subjects. In the simple correlation analysis, we used a threshold of $p<$ 0.005 , corresponding to $Z>2.80$, and cluster size $>40 \mathrm{~mm}^{3}$ for our predefined regions of interest (amygdala and piriform). For the rest of the brain, we used a more stringent criterion of an uncorrected $p<0.0005$ corresponding to $Z>3.48$ in combination with a cluster size $>40 \mathrm{~mm}^{3}$.

A psychophysiological interaction analysis (Friston et al., 1997; Heekeren et al., 2004) was also carried out. For each subject, we included the following in the linear regression model: (1) global signal variation resulting from physiological noises; (2) four hemodynamic response function-convolved regressors, each corresponding to an olfactory stimulus (task-related parameters, psychological factor); (3) the time series of the seed region (in this case the voxel with the peak $t$ value in the right fusiform with Talairach coordinates $35,-51,-7$ ) under each of the four olfactory conditions obtained as described in the simple correlation analysis section above (physiological signal); and (4) the product of (2) and (3) for each olfactory condition (hence psychophysiological interaction). The group analysis was performed as described above. If the connection of an area with the right fusiform region is unique to the sexual sweat condition, we would expect it to also significantly covary with the sexual psychophysiological interaction term.

\section{Results}

Subjects followed the breathing prompts and breathed constantly. There was no difference in either the breathing amplitude ( $p=0.602)$ or the frequency $(p=0.905)$ across the four olfactory conditions.

\section{Right OFC and right fusiform distinguished sexual sweat from PSP, neutral sweat, and the nonsocial control}

An omnibus $F$ test identified two regions that distinguished among the four olfactory conditions. One region was in the right OFC [Talairach coordinates 33, 40, -1 ; uncorrected $p<0.0005$, corresponding to $Z>3.48$, cluster size $=52 \mathrm{~mm}^{3}$, small volumecorrected (svc) $p<0.005$, peak $Z=3.898$ ] (Fig. 3a), and the other in the right fusiform (Talairach coordinates $35,-51,-7$; uncorrected $p<0.0005$, corresponding to $Z>3.48$, cluster size $=49$ $\mathrm{mm}^{3}$, peak $Z=4.542$ ) (Fig. $3 d$ ). Post hoc tests revealed that both regions significantly responded to the sexual sweat compared with the nonsocial baseline, and to the sexual sweat compared with either the neutral sweat or PSP ( $p<0.002$, Bonferroni corrected) (Fig. $3 b, c, e, f)$. Neither the right $\mathrm{OFC}$ nor the right fusiform region significantly differentiated among the nonsocial control, the neutral sweat, and PSP ( $p>0.9$, Bonferroni corrected $)$.

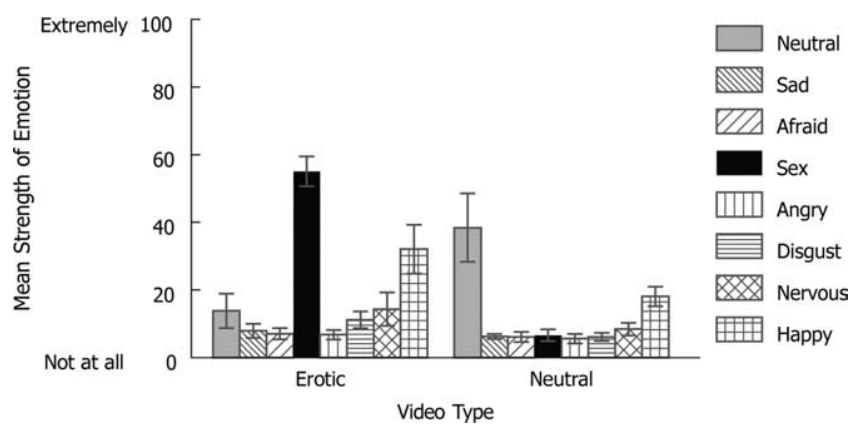

Figure 2. Mean strength of self-reported emotions from the three donors. This is shown on a $100 \mathrm{~mm}$ visual analog scale during the neutral and the erotic video segments, respectively. The plot shows that the emotion generation was successful. Neutral videos elicited predominantly neutral feelings. Erotic videos elicited predominantly sexual arousal and happiness. Error bars represent SEM.

Whereas OFC is a secondary olfactory region (Zatorre et al., 1992; Sobel et al., 2000; Zald and Pardo, 2000; Gottfried et al., 2002a; Anderson et al., 2003) (for a comprehensive review, see Gottfried and Zald, 2005), the activated fusiform region is not commonly associated with olfaction. Functional connectivity analysis (see Materials and Methods) yielded no significant connection between the right fusiform and the right OFC ( $p=0.76)$. Further analyses driven by the known functional anatomy of the olfactory system confirmed each of their HRs to be significantly correlated with the HRs of the primary olfactory area defined as the amygdala and the piriform (see supplemental Table S1, available at www.jneurosci.org as supplemental material). Additional search in the secondary olfactory areas identified that the HR of the right fusiform, but not the right OFC, was also significantly correlated with the HRs of the right thalamus (Talairach coordinates $25,-26,7 ; p<0.0005$, corresponding to $Z>3.48$, voxel size $=135 \mathrm{~mm}^{3}$, peak $\left.Z=4.166\right)$ and the right hippocampus/ parahippocampal gyrus (Talairach coordinates $30,-40,-1 ; p<$ 0.0005 , corresponding to $Z>3.48$, voxel size $=108 \mathrm{~mm}^{3}$, peak $Z=4.284)$. Whereas thalamus functions as a sensory relay, hippocampal/parahippocampal region has previously been shown to be involved in olfactory learning (Gottfried et al., 2002b) and olfactory memory (Gottfried et al., 2004). We thus infer that the olfactory input might have been relayed to the right fusiform activation via the primary olfactory areas, including the piriform and the amygdala, and the secondary olfactory areas such as the thalamus and the hippocampus/parahippocampal region.

Consistent with previous findings (Poellinger et al., 2001; Vuilleumier et al., 2004; Plailly et al., 2005), the involvement of the piriform, amygdala, and right thalamus, as well as the right hippocampal/parahippocampal region and right fusiform, is not specific to the task of smelling human sexual sweat. At $p=0.005$ corresponding to $Z=2.80$, we did not find any voxel in the brain whose HR uniquely covaries with the psychophysiological inter- 


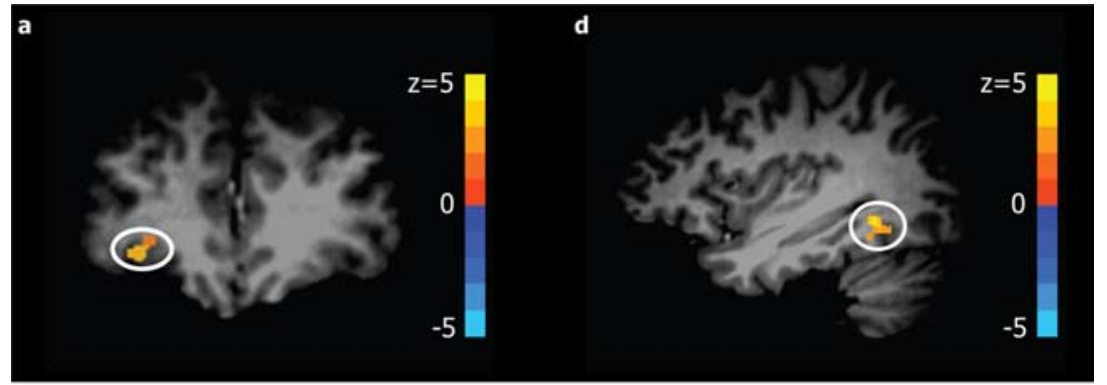

b

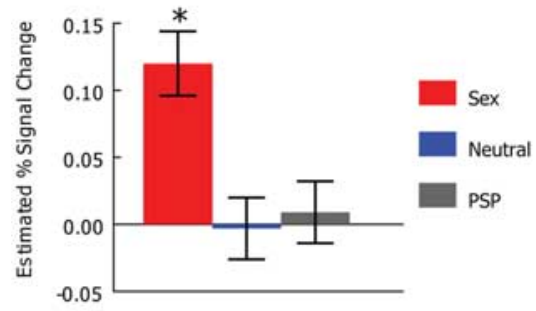

C
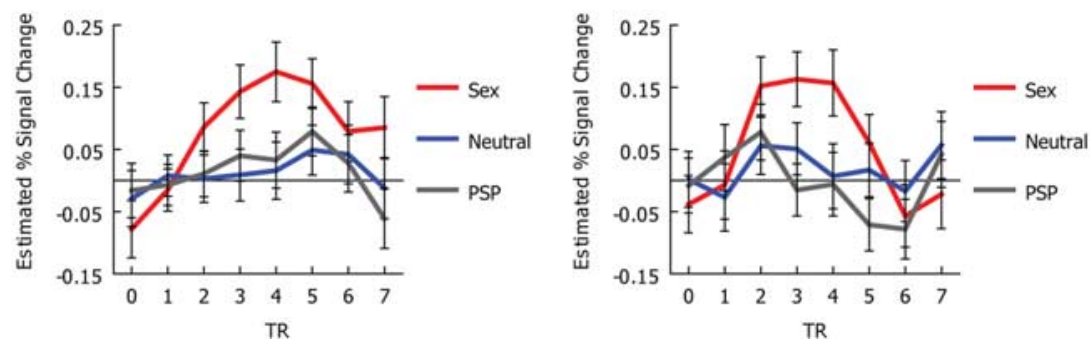

Figure 3. Brain responses to social chemosensory compounds. $\boldsymbol{a}$, Coronal view showing an area in the right orbitofrontal cortex ( 33,40 , -1) activated in the omnibus ANCOVAFtest (svc $p<0.005)$. $\boldsymbol{b}$, The means and SEs of the estimated percentage signal change in the right orbitofrontal region. $\boldsymbol{c}$, The time courses of the hemodynamic responses in the right orbitofrontal region. $\boldsymbol{d}$, Sagittal view showing a region in the right fusiform gyrus $(35,-51,-7)$ activated in the omnibus ANCOVA Ftest (uncorrected $p<0.0005$, cluster size $\left.=49 \mathrm{~mm}^{3}\right)$. $\boldsymbol{e}$ The means and SEs of the estimated percentage signal change in the right fusiform region. $f$, The time courses of the hemodynamic responses in the right fusiform region. In $\boldsymbol{a}$ and $\boldsymbol{d}$, the activation map (omnibus $F$ test) is overlaid on top of a high-resolution $\mathrm{T} 1$ image in Talairach space. In $\boldsymbol{c}$ and $\boldsymbol{f}, 0$ on the $\boldsymbol{x}$-axis refers to the onset of the olfactory stimuli. In $\boldsymbol{b}, \boldsymbol{c}, \boldsymbol{e}$, and $\boldsymbol{f}$, the response to PEA is used as the reference level. Sex, Sexual sweat; Neutral, neutral sweat. ${ }^{*} p<0.002$, Bonferroni corrected.
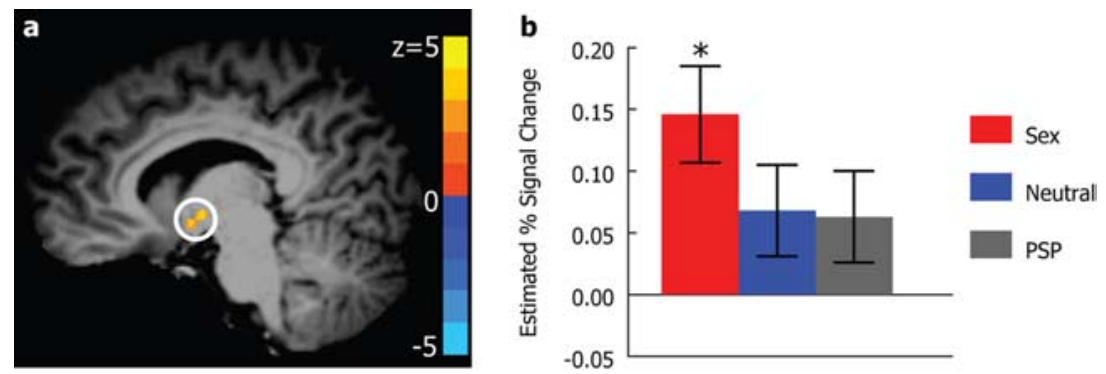

Figure 4. Right hypothalamic responses to social chemosensory compounds. $\boldsymbol{a}$, Sagittal view showing a region in the right hypothalamus $(8,-4,-2)$ that responded to the sexual sweat ( $\operatorname{svc} p<0.005)$. The activation map is overlaid on top of a high-resolution $\mathrm{T} 1$ image in Talairach space. $\boldsymbol{b}$, The means and SEs of the estimated percentage signal change in the right hypothalamic region. ${ }^{*} p<0.005$, Bonferroni corrected.

action term of the sexual sweat condition (see Materials and Methods).

\section{Hypothalamus differentiated sexual sweat from the nonsocial control}

Motivated by the extensive interest in the hypothalamus vis à vis sex and reproduction (Savic et al., 2001, 2005), we specifically examined whether the sexual sweat activated this area. We found that the right hypothalamus also significantly responded to the sexual sweat compared with the baseline nonsocial control $(\mathrm{Ta}$ lairach coordinates: $8,-4,-2$; uncorrected $p<0.005$, corresponding to $Z>$ 2.80 , cluster size $=59$, svc $p<0.005$, peak $Z=3.398$ ) (Fig. 4a), although there was no significant difference in the response among the social chemosensory compounds $(p>0.2$, Bonferroni corrected) (Fig. 4b).

\section{Discussion}

Because PSP at $250 \mu \mathrm{M}$ already heightens autonomic arousal in women (Jacob et al., 2001a; Bensafi et al., 2004), the lack of difference between our PSP sample $(916 \mu \mathrm{M})$ and the neutral sweat (containing negligible PSP; see Materials and Methods) in any of the aforementioned areas implied that the observed effects could not have been caused by autonomic arousal. This corroborates previous behavioral studies showing that the impact of social chemosignals (sweat from fearful or anxious individuals) on attention, person perception, and physiological responses is independent of mere arousal (Pause et al., 2004; Chen et al., 2006; Prehn et al., 2006).

Some previous PET studies reported hypothalamic response to PSP in heterosexual women (Savic et al., 2001) and gay men (Savic et al., 2005). PSP failed to reach significance in the current experiment $(p=0.092)$ (Fig. 4b). The discrepancy might be attributable to the differences in the methodology, but could also simply reflect differences in the PSP concentrations used [low (916 $\mu \mathrm{M}$ in propylene glycol) in the current study vs the maximal (pure crystal form of PSP) in the above (Savic et al., 2001, 2005) PET studies]. When we compared our findings with a PET study that used a PSP concentration more comparable to ours ( $250 \mu \mathrm{M}$ in propylene glycol with $1 \%$ clove oil) (Jacob et al., 2001b), and when we adopted the statistical threshold of that study (extent threshold $=240 \mathrm{~mm}^{3}, Z>2.71$ ) while still using the nonsocial smell as the baseline, we observed neural responses to PSP in regions consistent with the ones reported in that study, including the left precentral gyrus, left hippocampus, left cingulate, left cerebellum, and right medial frontal gyrus, as well as an area in the right basal ganglia overlapping with hypothalamus. To put our results in perspective, we note that the OFC is not only a secondary olfactory region, but also widely implicated in socioemotional regulation and behavior. It receives input from all the sensory modalities (Kringelbach, 2005) and has been proposed to participate in visual-olfactory learning (Got- 
tfried et al., 2002b; Gottfried and Dolan, 2004), mediation of hedonic experience (Kringelbach, 2005), reward monitoring (Gottfried and Dolan, 2004; Kringelbach, 2005), and emotion recognition (Adolphs, 2002). The hypothalamus is an olfactory region (Zatorre et al., 1992) (for a comprehensive review of the human olfactory cortex, see Gottfried, 2006) also known to be important in regulating sex and reproduction. Taking these into account, our results suggest that the right OFC, along with the right hypothalamus, processed the emotional value of the sexual sweat.

The right fusiform is well documented to hold modules specialized in human perception, namely, the fusiform face area (FFA) (Kanwisher et al., 1997) and the fusiform body area (FBA) (Schwarzlose et al., 2005). This region can be activated through both explicit (O'Craven and Kanwisher, 2000) and implicit (hearing familiar people's voices) (von Kriegstein et al., 2005) visual imageries. In addition, emotional stimuli augment its responses (Vuilleumier et al., 2001; Hadjikhani and de Gelder, 2003; Peelen et al., 2007). The Talairach coordinates of the fusiform region identified in our experiment fall in the range of the coordinates for FFA and FBA (Kanwisher et al., 1997; Schwarzlose et al., 2005). Such anatomical location likely reflects a recognition of the human quality in the sexual sweat, whose emotional nature may have also contributed to the activation. Considering its functional connectivity to the right hippocampus/parahippocampal gyrus, the recognition may arise from implicitly associating the sexual sweat with humans based on past experience. The fact that most subjects did not perceive the sexual sweat as human related (Table 1) suggests that the effects we observed occurred at a subconscious level.

The processing of human sexual sweat took place both within (OFC and hypothalamus) and beyond (fusiform) the olfactory system. Interestingly, with the exception of hypothalamus, neither the OFC nor the fusiform region is implicated in sexual motivation and behavior. Hence, our results implied that the chemosensory information from natural human sexual sweat was encoded more holistically in the brain rather than specifically for its sexual quality.

Finally, it is worth noting that the neural activity to airborne sexual sweat is lateralized to the right hemisphere. This observation is consistent with existing literature on the right hemispheric dominance in processing olfaction (Zatorre et al., 1992) (for a meta-analysis and review, see Gottfried and Zald, 2005), human social qualities (Kanwisher et al., 1997; Schwarzlose et al., 2005), and emotion (Cancelliere and Kertesz, 1990; Blonder et al., 1991).

Humans are evolved to respond to salient socioemotional information. Distinctive neural mechanisms underlie the processing of emotions in facial and vocal expressions. Our results here show that the brain also recognizes the socioemotional information contained in the human sexual sweat. This is so despite the facts that subjects were verbally unaware of the nature of the olfactory stimuli and that the intensity and pleasantness differences had been statistically controlled for. Our findings provide olfactory evidence that the right orbitofrontal cortex and the right fusiform participate in the processing of chemosensory human socioemotional information. Moreover, they also offer neural support for the existence of affective communication through human sociochemosensory cues, a subject extensively studied in animal research but hitherto little known in humans.

\section{References}

Adolphs R (2002) Neural systems for recognizing emotion. Curr Opin Neurobiol 12:169-177.

Anderson AK, Christoff K, Stappen I, Panitz D, Ghahremani DG, Glover G, Gabrieli JD, Sobel N (2003) Dissociated neural representations of intensity and valence in human olfaction. Nat Neurosci 6:196-202.

Bensafi M, Tsutsui T, Khan R, Levenson RW, Sobel N (2004) Sniffing a human sex-steroid derived compound affects mood and autonomic arousal in a dose-dependent manner. Psychoneuroendocrinology 29:1290-1299.

Blonder LX, Bowers D, Heilman KM (1991) The role of the right hemisphere in emotional communication. Brain 114:1115-1127.

Brand G, Millot JL (2001) Sex differences in human olfaction: between evidence and enigma. Q J Exp Psychol B 54:259-270.

Broday LR, Hall JA (2000) Gender, emotion, and expression. In: Handbook of emotions, Ed 2 (Lewis M, Haviland-Jones J, eds), pp 338-349. New York: Guilford.

Brooksbank BW, Wilson DA, MacSweeney DA (1972) Fate of androsta4,16-dien-3-one and the origin of 3-hydroxy-5-androst-16-ene in man. J Endocrinol 52:239-251.

Cancelliere AE, Kertesz A (1990) Lesion localization in acquired deficits of emotional expression and comprehension. Brain Cogn 13:133-147.

Chen D, Haviland-Jones J (2000) Human olfactory communication of emotion. Percept Mot Skills 91:771-781.

Chen D, Katdare A, Lucas N (2006) Chemosignals of fear enhance cognitive performance in humans. Chem Senses 31:415-423.

Cox RW (1996) AFNI: software for analysis and visualization of functional magnetic resonance neuroimages. Comput Biomed Res 29:162-173.

Friston KJ, Buechel C, Fink GR, Morris J, Rolls E, Dolan RJ (1997) Psychophysiological and modulatory interactions in neuroimaging. Neuroimage $6: 218-229$.

Gottfried JA (2006) Smell: central nervous processing. Adv Otorhinolaryngol 63:44-69.

Gottfried JA, Dolan RJ (2004) Human orbitofrontal cortex mediates extinction learning while accessing conditioned representations of value. Nat Neurosci 7:1144-1152.

Gottfried JA, Zald DH (2005) On the scent of human olfactory orbitofrontal cortex: meta-analysis and comparison to non-human primates. Brain Res Rev 50:287-304.

Gottfried JA, Deichmann R, Winston JS, Dolan RJ (2002a) Functional heterogeneity in human olfactory cortex: An event-related functional magnetic resonance imaging study. J Neurosci 22:10819-10828.

Gottfried JA, O’Doherty J, Dolan RJ (2002b) Appetitive and aversive olfactory learning in humans studied using event-related functional magnetic resonance imaging. J Neurosci 22:10829-10837.

Gottfried JA, Smith AP, Rugg MD, Dolan RJ (2004) Remembrance of odors past: human olfactory cortex in cross-modal recognition memory. Neuron 42:687-695.

Gower DB, Holland KT, Mallet AI, Rennie PJ, Watkins WJ (1994) Comparison of 16-androstene steroid concentrations in sterile apocrine sweat and axillary secretions: interconversions of 16 -androstenes by the axillary microflora-a mechanism for axillary odour production in man? J Steroid Biochem Mol Biol 48:409-418.

Gulyás B, Kéri S, O’Sullivan BT, Decety J, Roland PE (2004) The putative pheromone androstadienone activates cortical fields in the human brain related to social cognition. Neurochem Int 44:595-600.

Hadjikhani N, de Gelder B (2003) Seeing fearful body expressions activates the fusiform cortex and amygdala. Curr Biol 13:2201-2205.

Heekeren HR, Marrett S, Bandettini PA, Ungerleider LG (2004) A general mechanism for perceptual decision-making in the human brain. Nature 431:859-862.

Jacob S, McClintock MK (2000) Psychological state and mood effects of steroidal chemosignals in women and men. Horm Behav 37:57-78.

Jacob S, Hayreh DJS, McClintock MK (2001a) Context-dependent effects of steroid chemosignals on human physiology and mood. Physiol Behav 74:15-27.

Jacob S, Kinnunen LH, Metz J, Cooper M, McClintock MK (2001b) Sustained human chemosignal unconsciously alters brain function. Neuroreport 12:2391-2394.

Kanwisher N, McDermott J, Chun MM (1997) The fusiform face area: a module in human extrastriate cortex specialized for face perception. J Neurosci 17:4302-4311. 
Kringelbach ML (2005) The human orbitofrontal cortex: linking reward to hedonic experience. Nat Rev Neurosci 6:691-702.

Labows JN, Preti G (1992) Human semiochemicals. In: Fragrance: the psychology and biology of perfume (Van Toller S, Dodd GH, eds), pp 69-90. New York: Elsevier Science.

Lorig TS, Elmes DG, Zald DH, Pardo JV (1999) A computer-controlled olfactometer for fMRI and electrophysiological studies of olfaction. Behav Res Methods Instrum Comput 31:370-375.

Lundström JN, Gonçalves M, Esteves F, Olsson MJ (2003) Psychological effects of subthreshold exposure to the putative human pheromone 4,16androstadien-3-one. Horm Behav 44:395-401.

Lundström JN, Boyle JA, Zatorre RJ, Jones-Gotman M (2008) Functional neuronal processing of body odors differs from that of similar common odors. Cereb Cortex 18:1466-1474.

Mai JK, Assheuer J, Paxinos G (1997) Atlas of the human brain. San Diego: Academic.

O’Craven KM, Kanwisher N (2000) Mental imagery of faces and places activates corresponding stiimulus-specific brain regions. J Cogn Neurosci 12:1013-1023.

Pause BM, Ohrt A, Prehn A, Ferstl R (2004) Positive emotional priming of facial affect perception in females is diminished by chemosensory anxiety signals. Chem Senses 29:797-805.

Peelen MV, Atkinson AP, Andersson F, Vuilleumier P (2007) Emotional modulation of body-selective visual areas. Soc Cogn Affect Neurosci 2:274-283.

Plailly J, Bensafi M, Pachot-Clouard M, Delon-Martin C, Kareken DA, Rouby C, Segebarth C, Royet JP (2005) Involvement of right piriform cortex in olfactory familiarity judgments. Neuroimage 24:1032-1041.

Poellinger A, Thomas R, Lio P, Lee A, Makris N, Rosen BR, Kwong KK (2001) Activation and habituation in olfaction-an fmri study. Neuroimage 13:547-560.

Prehn A, Ohrt A, Sojka B, Ferstl R, Pause BM (2006) Chemosensory anxiety signals augment the startle reflex in humans. Neurosci Lett 394:127-130.

Russell MJ (1976) Human olfactory communication. Nature 260:520-522.

Savic I, Berglund H, Gulyas B, Roland P (2001) Smelling of odorous sex hormone-like compounds causes sex-differentiated hypothalamic activations in humans. Neuron 31:661-668.

Savic I, Berglund H, Lindström P (2005) Brain response to putative pheromones in homosexual men. Proc Natl Acad Sci U S A 102:7356-7361.

Schwarzlose RF, Baker CI, Kanwisher N (2005) Separate face and body selectivity on the fusiform gyrus. J Neurosci 25:11055-11059.
Sobel N, Prabhakaran V, Desmond JE, Glover GH, Sullivan EV, Gabrieli JD (1997) A method for functional resonance imaging of olfaction. J Neurosci Methods 78:115-123.

Sobel N, Prabhakaran V, Zhao Z, Desmond JE, Glover GH, Sullivan EV, Gabrieli JD (2000) Time course of odorant-induced activation in the human primary olfactory cortex. J Neurophysiol 83:537-551.

Stables LA, Kennan RP, Gore JC (1998) Asymmetric spin-echo imaging of magnetically inhomogeneous systems: theory, experiment, and numerical studies. Magn Reson Med 40:432-442.

Stern K, McClintock MK (1998) Regulation of ovulation by human pheromones. Nature 392:177-179.

Talairach J, Tournoux P (1988) Co-planar stereotaxic atlas of the human brain: 3-dimensional proportional system: an approach to cerebral imaging. Stuttgart, Germany: Thieme.

von Kriegstein K, Kleinschmidt A, Sterzer P, Giraud AL (2005) Interaction of face and voice areas during speaker recognition. J Cogn Neurosci 17:367-376.

Vuilleumier P, Armony JL, Driver J, Dolan RJ (2001) Effects of attention and emotion on face processing in the human brain: an event-related fmri study. Neuron 30:829-841.

Vuilleumier P, Richardson MP, Armony JL, Driver J, Dolan RJ (2004) Distant influences of amygdala lesion on visual cortical activation during emotional face processing. Nat Neurosci 7:1271-1278.

Ward BD (2000) Simultaneous inference for fmri data. In: Afni 3ddeconvolve documentation. Milwaukee, WI: Medical College of Wisconsin.

Wyart C, Webster WW, Chen JH, Wilson SR, McClary A, Khan RM, Sobel N (2007) Smelling a single component of male sweat alters levels of cortisol in women. J Neurosci 27:1261-1265.

Wyatt TD (2003) Pheromones and animal behavior: communication by smell and taste. London: Cambridge UP.

Zald DH, Pardo JV (2000) Functional neuroimaging of the olfactory system in humans. Int J Psychophysiol 36:165-181.

Zatorre RJ, Jones-Gotman M, Evans AC, Meyer E (1992) Functional localization and lateralization of human olfactory cortex. Nature 360:339-340.

Zeng XN, Leyden JJ, Lawley HJ, Sawano K, Nohara I, Preti G (1991) Analysis of characteristic odors from human male axillae. J Chem Ecol 17:1469-1492.

Zhou W, Chen D (2009) Fear-related chemosignal modulates fear recognition in ambiguous facial expressions. Psychol Sci, in press. 